\title{
Left atrial 4D flow cardiovascular magnetic resonance: a reproducibility study in sinus rhythm and atrial fibrillation
}

\author{
Marco Spartera ${ }^{1,2^{*}} \mathbb{D}$, Guilherme Pessoa-Amorim ${ }^{1,2}$, Antonio Stracquadanio ${ }^{1,2}$, Adam Von Ende , \\ Alison Fletcher ${ }^{3}$, Peter Manley ${ }^{3}$, Stefan Neubauer ${ }^{1,2}$, Vanessa M. Ferreira ${ }^{1,2}$, Barbara Casadei ${ }^{1}$, Aaron T. Hess $s^{1,2+}$ \\ and Rohan S. Wijesurendra ${ }^{1,2+}$
}

\begin{abstract}
Background: Four-dimensional (4D) flow cardiovascular magnetic resonance (CMR) allows sophisticated quantification of left atrial (LA) blood flow, and could yield novel biomarkers of propensity for intra-cardiac thrombus formation and embolic stroke. As reproducibility is critically important to diagnostic performance, we systematically investigated technical and temporal variation of LA 4D flow in atrial fibrillation (AF) and sinus rhythm (SR).

Methods: Eighty-six subjects $(S R, n=64 ; A F, n=22)$ with wide-ranging stroke risk $\left(\mathrm{CHA}_{2} \mathrm{DS}_{2}\right.$ VASC $\left.0-6\right)$ underwent LA 4D flow assessment of peak and mean velocity, vorticity, vortex volume, and stasis. Eighty-five (99\%) underwent a second acquisition within the same session, and 74 (86\%) also returned at 30 (27-35) days for an interval scan. We assessed variability attributable to manual contouring (intra- and inter-observer), and subject repositioning and reacquisition of data, both within the same session (same-day scan-rescan), and over time (interval scan). Within-subject coefficients of variation (CV) and bootstrapped $95 \% \mathrm{Cls}$ were calculated and compared.
\end{abstract}

Results: Same-day scan-rescan CVs were 6\% for peak velocity, 5\% for mean velocity, 7\% for vorticity, 9\% for vortex volume, and $10 \%$ for stasis, and were similar between SR and AF subjects (all $p>0.05$ ). Interval-scan variability was similar to same-day scan-rescan variability for peak velocity, vorticity, and vortex volume (all $p>0.05$ ), and higher for stasis and mean velocity (interval scan CVs of $14 \%$ and $8 \%$, respectively, both $\mathrm{p}<0.05$ ). Longitudinal changes in heart rate and blood pressure at the interval scan in the same subjects were associated with significantly higher variability for LA stasis $(p=0.024)$, but not for the remaining flow parameters (all $p>0.05$ ). SR subjects showed significantly greater interval-scan variability than AF patients for mean velocity, vortex volume, and stasis (all $p<0.05$ ), but not peak velocity or vorticity (both $p>0.05$ ).

Conclusions: LA peak velocity and vorticity are the most reproducible and temporally stable novel LA 4D flow biomarkers, and are robust to changes in heart rate, blood pressure, and differences in heart rhythm.

Keywords: Left atrium, 4D flow, Stasis, Velocities, Vorticity, Vortex, Reproducibility, Cardioembolic risk, Cardiovascular magnetic resonance

\footnotetext{
*Correspondence: marco.spartera@gmail.com

${ }^{\dagger}$ Aaron T. Hess and Rohan S. Wijesurendra contributed equally to this work

${ }^{1}$ Division of Cardiovascular Medicine, Radcliffe Department of Medicine, John Radcliffe Hospital, University of Oxford, West Wing, Headley Way, Oxford, UK

Full list of author information is available at the end of the article
}

\section{Introduction}

Stroke is the second most common cause of death and the third most common cause of disability worldwide [1]. Atrial fibrillation (AF) is a strong risk factor for embolic stroke mainly as it causes pro-thrombotic alterations in atrial blood flow and consequent thrombus formation original author(s) and the source, provide a link to the Creative Commons licence, and indicate if changes were made. The images or other third party material in this article are included in the article's Creative Commons licence, unless indicated otherwise in a credit line to the material. If material is not included in the article's Creative Commons licence and your intended use is not permitted by statutory regulation or exceeds the permitted use, you will need to obtain permission directly from the copyright holder. To view a copy of this licence, visit http://creativecommons.org/licenses/by/4.0/. The Creative Commons Public Domain Dedication waiver (http://creativeco mmons.org/publicdomain/zero/1.0/) applies to the data made available in this article, unless otherwise stated in a credit line to the data. 
within the left atrium (LA) [2-5]. Pooled evidence from echocardiography, autopsies, and surgical inspection of the LA reveals that intracardiac thrombus formation generally occurs within the left atrial appendage (LAA), particularly in non-rheumatic heart disease $[6,7]$. Overall, as many as $40 \%$ of all ischaemic strokes may be due to embolism from the LAA, encompassing not only patients with AF prior to or at the time of the stroke, but also those with AF detected sub-clinically on subsequent cardiac monitoring, as well as a proportion of patients with embolic stroke of unknown origins with signs of atrial myopathy [8].

Prediction of ischaemic stroke risk in clinical practice relies almost exclusively on scoring systems based on upstream clinical risk factors, such as the $\mathrm{CHA}_{2} \mathrm{DS}_{2}$ VASc score [9], which apportions one point each for heart failure $(\mathrm{C})$, hypertension $(\mathrm{H})$, age $\geq 65$ years $(\mathrm{A})$, age $\geq 75$ years (a further point, i.e. A2), diabetes (D), prior thromboembolic disease (S2, two points), vascular disease (VA), and female sex category (Sc). Although higher scores are linked to higher risk of incident ischaemic stroke, this and other similar clinical prediction tools are limited by a modest predictive capacity both in AF and in sinus rhythm (SR) patients $[9,10]$. Hence, the ability to assess parameters such as LA blood flow characteristics comprehensively (i.e. absolute flow velocities, the degree of stasis, and vortical flow patterns) both in AF and in SR is very attractive, since these features contribute directly to the downstream pathophysiologic substrate for thrombus formation and may be mechanistically relevant to the risk of embolic stroke [2].

ECG-gated time-resolved phase-contrast cardiovascular magnetic resonance (CMR) with 3-directional velocity encoding (4D flow) is an established CMR technique that allows comprehensive in-vivo visualisation and quantification of cardiovascular flow velocities [11]. Recent proof-of-principle studies have shown that peak and mean velocity and stasis measurements derived from LA 4D flow are associated with clinical stroke risk in patients in SR with history of AF $[12,13]$ and low blood flow velocities have been linked to activation of the coagulation cascade $[2,3]$. Furthermore, assessment of the pattern of blood flow within the LA [14] may also be relevant to embolic risk, since loss of normal vortical flow has been found in patients in SR with a history of paroxysmal AF at higher stroke risk [13] and is associated with platelet aggregation and thrombus formation $[15,16]$. Together, these observations raise the intriguing possibility that parameters derived from 4D flow could eventually be used as imaging biomarkers to improve embolic risk stratification in clinical practice, particularly in patients with SR. Nevertheless, it is currently unclear whether LA 4D flow parameters can be measured in a reproducible fashion. Hence, the first step to clinical application is the systematic evaluation of reproducibility and temporal variation, as these directly impact on diagnostic performance and hence the feasibility and power calculations for larger-scale studies. Although analysis of LA 4D flow parameters by CMR is more time-consuming than the collection of simpler measurements, such as LA volume and emptying fraction, direct assessment of a key component of the Virchow's triad would be highly attractive if able to identify patients who may benefit from anticoagulation, either in SR or with a low AF burden (e.g., not identified by short-term electrocardiographic (ECG) monitoring [17]). In the present study, we aimed to evaluate and compare the reproducibility of a number of LA flow characteristic parameters $[12,13]$, both within the same session and over time. We included subjects with $\mathrm{AF}$ or SR and a wide range of clinical stroke risk, to provide information on reproducibility across a heterogeneous and clinically-relevant population.

\section{Methods}

This study was undertaken in a single tertiary centre (University of Oxford, Radcliffe Department of Medicine, Oxford, UK). The study protocol was approved by a local Research Ethics Committee, and all patients gave written informed consent.

\section{Design}

Patients (in AF or SR) were prospectively non-consecutively recruited from a number of sources at our institution, including outpatient clinics, the waiting list for electrical cardioversion, clinical CMR referrals, a pool of control subjects who had participated in other research studies, and through word of mouth. Young healthy subjects in SR $(\mathrm{N}=14,16 \%)$ were recruited under an ethically approved departmental technical development protocol and provided verbal consent (as specified in the protocol).

Subjects were recruited with a wide range of clinical stroke risk, as assessed by the $\mathrm{CHA}_{2} \mathrm{DS}_{2}$ VASc score [9]. Patient medical records were screened to document stroke risk factors where available, and a research questionnaire designed to collect this information was administered to all participants.

\section{CMR imaging protocol}

CMR examinations were performed by one of three operators (AF, PM, or MS) on one of two 3T CMR systems (Verio syngo MR B17 and MAGNETOM Prisma VE11C, both Siemens Healthineers, Erlangen, Germany).

At the baseline scan, the CMR protocol included retrospectively ECG-gated time-resolved balanced steadystate free precession (bSSFP) cine imaging in horizontal 
long axis ('four-chamber') and vertical long-axis ('twochamber'), for the evaluation of left ventricular (LV) ejection fraction (LVEF) and volumes, and LA emptying function (LAEF) and volumes as previously described $[18,19]$. All images were analysed using cvi42 software (version 5.3.4, Circle Cardiovascular Imaging Inc, Calgary, Alberta, Canada).

Additionally, the 4D flow sequence was undertaken twice during the same baseline session with removal of the subject from the scanner and performing repeat isocentre positioning (scan 1a and scan 1b) for determination of 'same-day scan-rescan' variability. The same 4D flow sequence was also undertaken again at an interval of approximately 1 month in the same scanner (scan 2) for determination of 'interval scan-rescan' variability. In 4 subjects (5\%) in whom 'same-day scan-rescan' variability had not been determined at scan 1 , this was done during scan 2 instead. The 4D flow protocol included retrospectively ECG-gated time-resolved 3D phase-contrast CMR with 3-directional velocity encoding (' $4 \mathrm{D}$ flow') imaging to allow in vivo assessment of blood flow velocities in the LA. In two cases (2\%), a prospectively triggered $4 \mathrm{D}$ flow sequence was used instead. The 4D flow CMR data were acquired during free breathing using navigator gating of diaphragmatic motion, with pulse sequence parameters as follows: flip angle $=6-7^{\circ}$, spatial resolution/voxel size $2.4 \times 2.4 \times 2.5-3.0 \times 3.0 \times 3.0 \mathrm{~mm}$, temporal resolution $=40-49 \mathrm{~ms}$, reconstructed to $20-30$ time frames, $\mathrm{TE}=2.39-3.66 \mathrm{~ms}$, imaging acceleration (GRAPPA technique) with an acceleration factor of $\mathrm{R}=3$, total acquisition time $=5-20$ min depending on heart rate and navigator efficiency, velocity encoding sensitivity $=110-120 \mathrm{~cm} / \mathrm{s}$. The field-of-view (FOV) was axial and adjusted to encompass the entire LA in each subject. Further details of CMR protocols is available in Additional file 1: methods.

\section{LA 4D flow processing}

All 4D flow were acquired with Maxwell term correction [20], DICOM tools from the OXSA toolbox were used [21], and data were corrected for velocity aliasing [22], and eddy currents [23] using a Matlab-based in-house software designed by ATH and MS (MATLAB R2015 8.6.0.267246; Mathworks, Natick, Massachusetts, USA), available on the Oxford University Research Archive (ORA) platform (https://doi.org/10.5287/bodleian:ey4ov $\mathrm{zdbB}$ ). A three-dimensional time-averaged phase contrast angiogram was generated to facilitate better orientation in the context of the LA anatomy and interpretation of the flow data [24]. To isolate the LA velocity data, data analysis included 3D segmentation of the minimum LA volume (directly on magnitude images in the 4D flow dataset) which was obtained using cvi42 software.
Two sets of parameters were derived from 4D flow dataset: (A) absolute velocities and (B) directionality of flow and LA flow patterns.

The absolute atrial velocities for each voxel inside the segmented LA were determined as described previously [12] (see Fig. 1). Briefly, for each voxel, the peak and mean velocities $(\mathrm{m} / \mathrm{s})$ were determined; stasis was calculated as the ratio of the total number of cardiac time frames (n) with a velocity below the threshold of $0.1 \mathrm{~m} / \mathrm{s}$ ( $\mathrm{n}$ stasis) to the total number of frames (NTot) $[12,25]$ : stasis $=\frac{\text { nstasis }}{\text { NTot }}$. Global LA stasis was defined as the average proportion of stasis across all LA voxels and it is expressed as a proportion from 0 to 1 , where 0 is the lowest amount of stasis and 1 is the highest $[12,25]$.

Regarding flow patterns, a 4D velocity field within the LA was used to generate 3D pathlines and to evaluate atrial flow patterns during both LV systole and diastole using the Runge-Kutta method [26, 27] with Ensight software (2019 R1, ANSYS, Inc., Canonsburg, Pennsylvania, USA), see Fig. 1. Pathlines were emitted at each time-frame from probe planes positioned at each of the pulmonary veins (starting at the beginning of the cardiac cycle), allowing derivation of flow patterns inside the LA during both ventricular systole and diastole (based on mitral valve opening time derived from the magnitude images). Flow patterns were evaluated after quality control steps were applied, as previously described [28]. Normal flow within the LA is characterized by the presence of a vortex $[14,26,29]$ (i.e. pathlines with a circular/elliptic trajectory see Fig. 1). Atypical flow patterns observed were classified visually into short-ranged vortex (pathlines failing to reach the mitral valve plane during one averaged cardiac cycle, see Fig. 1), and into other patterns. Further, for all scans, after flow denoising using divergence free wavelets [30], the volumes of the largest vortex rings inside the LA were calculated automatically using the lambda2 $(\lambda 2)$ method [26]. In each case, a specific lambda threshold for vortices was defined as half the mean $\lambda 2$ value over the cardiac cycle [13]. LA vortex volume $(\mathrm{ml})$ is reported as the mean of LA vortex volumes throughout the cardiac cycle alongside peak LA vortex volumes $(\mathrm{ml})$ within systole and diastole. Finally, we also applied a measure of tendency of the blood flow to rotate using the volume-averaged magnitude of vorticity (units radians/s) [31, 32], after flow denoising [30]. LA vorticity is reported as the area under the vorticity-time curve over the entire cardiac cycle (in radians or rad), see Fig. 1.

\section{Blinding}

All imaging assessments (including LA and LV function, 4D flow parameters, and flow visualization data) for all the scans (including scan 1a, 1b, and 2) were conducted by investigators blinded to all clinical information 


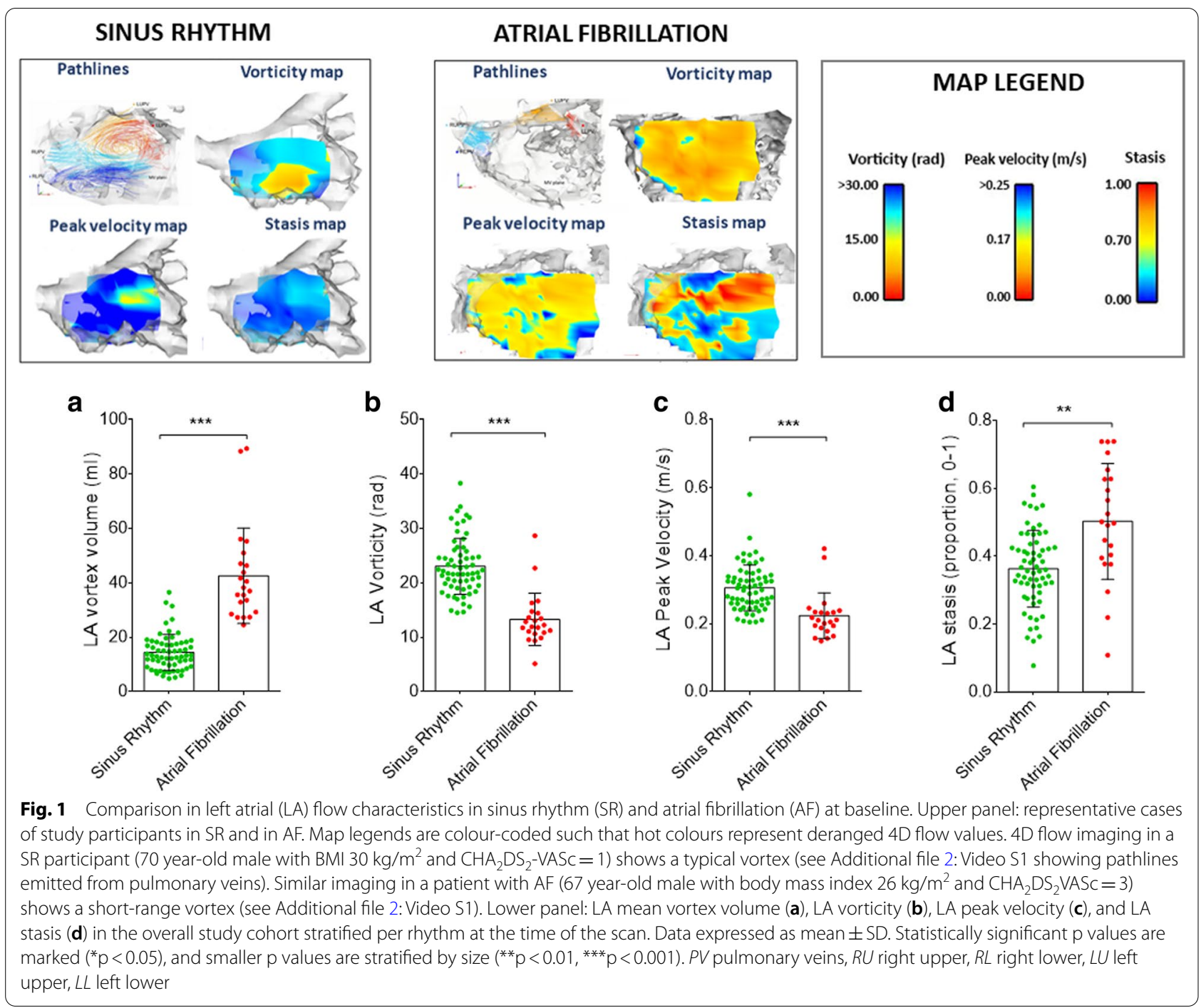

including rhythm at the time of the scan. After appropriate training, a single observer (GPA) contoured all cine imaging and LA 4D Flow sequence, according to a dedicated in-house post-processing protocol. A single observer (AS) processed all the qualitative data regarding flow patterns using the Ensight software, according to a separate dedicated in-house post-processing protocol. To evaluate intra-observer variability, the scans were re-contoured by the same observer (GPA) after an interval of at least 2 weeks. Inter-observer variability was conducted independently by a second independent observer (MS) according to the same post-processing protocol, and the 2 observers were blinded to each other. In order to ensure that the observers of scan 1a were blinded to the results of their own analysis of scan 1a and 1b (for intra-/interobserver $\mathrm{CV}$ and rescan $\mathrm{CV}$ ), the contours performed by GPA/MS were then imported into our MATLAB software (Mathworks) independently by AS who produced the MATLAB output with the results. Finally, to evaluate interval-scan $\mathrm{CV}$, scan 1 and scan 2 were anonymized with a different code in a way that it was impossible to ascertain that scan 1 and 2 belonged to the same research participant.

\section{Statistical analysis}

Data were examined for normality by visual inspection of histograms and Q-Q plots, and for homoscedasticity using Levene's test. Data are shown as mean \pm SD if normally distributed, or median (Q1-Q3) if non-normally distributed, or number (percentage), unless otherwise specified.

To quantify reproducibility and temporal variability, the following metrics were used: Bland-Altman limits of agreement and the within-subject coefficient of variation (CV) [33], which was calculated using the root mean square method and is reported as a proportion (from 0 
to 1). Bootstrap resampling (1000 samples) was used to calculate a non-parametric $95 \%$ confidence interval (CI) for the CV [33], as well as to estimate $95 \%$ CIs and p-values for the difference in $\mathrm{CV}$ values between independent groups (i.e. AF vs. SR patients; subjects with longitudinal change $\geq 10 \mathrm{mmHg}$ vs. $<10 \mathrm{mmHg}$ in blood pressure; and subjects with longitudinal change $\geq 10 \mathrm{bpm}$ vs. $<10 \mathrm{bpm}$ in heart rate, where changes are calculated from scan 1a to scan 2). A similar resampling strategy (10,000 bootstrap samples) was used to test differences between dependent CVs (e.g. scan-rescan vs. scan-interval scan). Further, the absolute agreement of the LA flow characteristics values obtained by the two independent observers (i.e. inter-rater variability) was assessed using the intraclass correlation coefficient (ICC) with a two-way mixed model.

Normally-distributed baseline data with equal variances across two independent groups (e.g. AF vs. SR) were compared using the unpaired t-test, and Welch's tests for normal data with unequal variances. Non-normally distributed unpaired baseline data were compared using the Mann-Whitney $\mathrm{U}$ test. Categorical baseline data were compared by using the Chi square or Fischer's exact tests (if cell size $<5$ ). Flow patterns from related measures were compared with McNemar's test. Intercorrelations among flow parameters were calculated using Pearson's coefficient with 1000 bootstraps 95\% CI (with sampling stratification for rhythm at the time of the scan).

All tests were 2-tailed, and values of $p<0.05$ were considered significant. All $\mathrm{p}$ values from pairwise comparisons were adjusted using the Bonferroni method.

We calculated that recruitment of a minimum of 74 subjects would provide $90 \%$ power (with two-tailed 0.05 type I error) to detect a 0.01 point mean difference in LA stasis between scan and rescan and between scan and interval scan, with an expected standard deviation of differences of 0.05 and pre-defined clinical agreement limit of 0.15 (based on pilot data).

Statistical analyses were performed with SPSS Statistics for Windows (version 25.0, Statistical Package for the Social Sciences, International Business Machines, Inc., Armonk, New York, USA), R 3.6.3 for computation of CV and bootstrapped 95\% CI, GraphPad Prism (version 6.01, GraphPad Software, San Diego, California, USA) for the figures and graphs, and MedCalc Version 19.1.3 (MedCalc Software, Mariakerke, Belgium) for power calculations.

\section{Results}

A total of 86 participants were included: 64 in SR at the time of the CMR scan, and 22 in AF at the time of the CMR scan. Baseline characteristics of all subjects are summarised in Additional file 1: Table S1. As expected, AF patients had profoundly altered LA flow characteristics compared to subjects in SR, as characterised by greater stasis, lower peak velocity, lower vorticity, and larger vortex size (all $\mathrm{p} \leq 0.001$; Fig. 1 ), as well as changes in other LA flow measurements (Additional file 1: Table S2). Exploratory analyses assessing LA 4D Flow parameters by age and gender, and their correlation with clinical characteristics are reported in Additional file 1: Tables S3, S4.

A total of 84 baseline scans (SR, $\mathrm{n}=62 ; \mathrm{AF}, \mathrm{n}=22$ ) were re-contoured and re-analysed by the same blinded investigator, while 75 baseline scans $(\mathrm{SR}, \mathrm{n}=53$; $\mathrm{AF}$, $\mathrm{n}=22$ ) were also re-contoured and re-analysed by a second blinded investigator. A total of 85 participants (SR, $\mathrm{n}=63$; $\mathrm{AF}, \mathrm{n}=22$ ) underwent the same 4D Flow sequence twice during the same visit with removal from the scanner and re-positioning for assessment of sameday scan-rescan variability. After an interval of 30 (2735 ) days, the scan was repeated ('scan 2') in 74 subjects ( $\mathrm{SR}, \mathrm{n}=56$; $\mathrm{AF}, \mathrm{n}=18$ ) for assessment of interval scanrescan variability. All subjects scanned in SR at scan 1 were in SR at scan 2, and all subjects scanned in AF at scan 1 remained in AF at scan 2.

\section{Reproducibility and temporal variability in the whole cohort}

Intra-observer and inter-observer CVs ranged from 1 to $3 \%$ and from 4 to $8 \%$, respectively, for LA mean and peak velocity, and stasis (Table 1; Fig. 2), but were larger for vortex volume, which displayed an intra-observer $\mathrm{CV}$ of $7.6 \%$ and an inter-observer $\mathrm{CV}$ of $11.2 \%$. Inter-observer variability was excellent for all parameters (ICC $\geq 0.91$, all $\mathrm{p}<0.001$, Additional file 1: Table S5).

Scan-rescan variability ranged between 5 and $7 \%$ for all LA flow parameters, except for vortex volume and stasis (CVs of $9 \%$ and $10 \%$, respectively).

Interval-scan variability ranged from 7\% (for peak velocity and vorticity) to $14 \%$ (for stasis, Table 1; Fig. 2).

Bland-Altman plots are presented in Additional file 1: Fig. S1 (for intra- and inter-observer measurements) and in Additional file 1: Fig. S2 (for rescan and interval scan measurements), with the corresponding mean bias and 95\% limits of agreement (LoA) reported in Table 1 . Bland-Altman plots revealed no likely proportional bias for any parameter except LA vortex volume which displayed a wider spread at higher values in the interval scan (Table 1 and Additional file 1: Fig. S2).

Analyses of the reproducibility and temporal variability of qualitative flow patterns assessed by means of pathlines are presented in Table 2 . There were no significant paired changes in flow patterns between scan and rescan 
Table 1 Reproducibility in the whole cohort

\begin{tabular}{|c|c|c|c|c|c|c|c|c|}
\hline & 1 & 2 & 3 & 4 & & & & \\
\hline & $\begin{array}{l}\text { Intra-observer } \\
\mathrm{N}=84\end{array}$ & $\begin{array}{l}\text { Inter-observer } \\
N=75\end{array}$ & $\begin{array}{l}\text { Same-day } \\
\text { rescan } \\
N=85\end{array}$ & $\begin{array}{l}\text { Interval Scan } \\
N=74\end{array}$ & p value (1 vs. 2) & p value (1 vs. 3) & p value (2 vs. 3 ) & $\begin{array}{l}p \text { value (3 } \\
\text { vs. } 4 \text { ) }\end{array}$ \\
\hline \multicolumn{9}{|l|}{ LA stasis, \% } \\
\hline CV $(95 \% \mathrm{Cl})$ & $\begin{array}{l}0.032(0.023- \\
0.041)\end{array}$ & $\begin{array}{l}0.073(0.052- \\
0.097)\end{array}$ & $\begin{array}{l}0.101 \text { (0.080- } \\
0.124)\end{array}$ & $\begin{array}{l}0.144(0.116- \\
0.175)\end{array}$ & $<0.001$ & $<0.001$ & 0.076 & 0.015 \\
\hline Bias (BA limits) & $\begin{array}{l}0.01(-0.02 \text { tc } \\
0.02)\end{array}$ & $\begin{array}{l}0.00(-0.09 \text { tc } \\
0.07)\end{array}$ & $\begin{array}{l}0.00(-0.09 t \\
0.09)\end{array}$ & $\begin{array}{l}0-0.01(-0.16 \\
\quad \text { to } 0.14)\end{array}$ & & & & \\
\hline \multicolumn{9}{|l|}{$\begin{array}{l}\text { LA peak veloc- } \\
\text { ity, } \mathrm{m} / \mathrm{s}\end{array}$} \\
\hline CV $(95 \%$ Cl) & $\begin{array}{l}0.012(0.009- \\
0.015)\end{array}$ & $\begin{array}{l}0.080(0.039- \\
0.131)\end{array}$ & $\begin{array}{l}0.065(0.042- \\
0.093)\end{array}$ & $\begin{array}{l}0.068(0.058- \\
0.079)\end{array}$ & 0.007 & $<0.001$ & 0.645 & 0.795 \\
\hline Bias (BA limits) & $\begin{array}{l}0.00(-0.01 \text { tc } \\
0.01)\end{array}$ & $\begin{array}{l}0.01(-0.09 \text { to } \\
0.07)\end{array}$ & $\begin{array}{l}0.00(-0.05 t \\
0.06)\end{array}$ & $\begin{array}{l}0.00(-0.05 \text { to } \\
0.06)\end{array}$ & & & & \\
\hline \multicolumn{9}{|c|}{$\begin{array}{l}\text { LA mean veloc- } \\
\text { ity, } \mathrm{m} / \mathrm{s}\end{array}$} \\
\hline CV $(95 \%$ Cl) & $\begin{array}{l}0.010(0.008- \\
0.013)\end{array}$ & $\begin{array}{l}0.037(0.025- \\
0.051)\end{array}$ & $\begin{array}{l}0.051(0.038- \\
0.064)\end{array}$ & $\begin{array}{l}0.076(0.063- \\
0.088)\end{array}$ & $<0.001$ & $<0.001$ & 0.134 & 0.007 \\
\hline Bias (BA limits) & $\begin{array}{l}0.00(0.00 \text { to } \\
0.00)\end{array}$ & $\begin{array}{l}0.00(-0.01 \text { to } \\
0.01)\end{array}$ & $0.00(-0.020 t$ & $\begin{array}{l}0.00(-0.03 \text { to } \\
0.03)\end{array}$ & & & & \\
\hline \multicolumn{9}{|l|}{ LA vorticity, rad } \\
\hline CV $(95 \%$ Cl) & $\begin{array}{l}0.012(0.010- \\
0.014)\end{array}$ & $\begin{array}{l}0.081(0.041- \\
0.126)\end{array}$ & $\begin{array}{l}0.072(0.052- \\
0.094)\end{array}$ & $\begin{array}{l}0.074(0.059- \\
0.090)\end{array}$ & 0.002 & $<0.001$ & 0.816 & 0.875 \\
\hline Bias (BA limits) & $\begin{array}{l}0.00(-0.73 \text { tc } \\
0.73)\end{array}$ & $\begin{array}{l}0-0.17(-4.67 \\
\text { to } 4.32)\end{array}$ & $\begin{array}{l}-0.01(-4.12 \\
\text { to } 4.15)\end{array}$ & $\begin{array}{l}0.41(-3.90 \text { to } \\
4.73)\end{array}$ & & & & \\
\hline \multicolumn{9}{|l|}{$\begin{array}{l}\text { LA vortex } \\
\text { volume, ml }\end{array}$} \\
\hline CV $(95 \%$ Cl) & $\begin{array}{l}0.076(0.053- \\
0.099)\end{array}$ & $\begin{array}{l}0.112(0.083- \\
0.143)\end{array}$ & $\begin{array}{l}0.086(0.059- \\
0.115)\end{array}$ & $\begin{array}{l}0.108(0.080- \\
0.137)\end{array}$ & 0.045 & 0.564 & 0.172 & 0.254 \\
\hline Bias (BA limits) & $\begin{array}{l}-1.12(-5.44 \\
\text { to } 3.05)\end{array}$ & $\begin{array}{l}-1.18(-6.45 \\
\text { to } 4.10)\end{array}$ & $\begin{array}{l}0.42(-3.22 t \\
4.06)\end{array}$ & $\begin{array}{c}0-0.51(-7.32 \\
\text { to } 60.30)\end{array}$ & & & & \\
\hline
\end{tabular}

Within-subject coefficients of variation (CV, reported as proportion) are presented with bootstrapped $95 \%$ Cls (1000 bootstraps). Mean bias and Bland-Altman (BA) $95 \%$ limits of agreement are also reported. $p$ values are obtained by bootstrapped differences (10,000 bootstraps) between CV values for each parameter. Significant $p$ values $(<0.05)$ are shown in italic

LA left atrial

and no significant changes over time in the interval scans (all p > 0.05; Table 2).

\section{Assessment of sources of variability in LA flow characteristics}

In order to assess the sources of variability, comparison among CVs is reported for all flow parameters in Table 1; Fig. 2.

All flow parameters showed statistically significant differences in variability due to manual segmentation by two independent observers (intra-observer vs. inter-observer $\mathrm{CV}$ differences from 3 to $7 \%$, all $\mathrm{p}<0.05$, Table 1; Fig. 2). By contrast, we found no significant differences in variability related to the rescan (i.e., to repositioning and reacquisition within the same session) over inter-observer variability for any of the parameters evaluated $(p>0.05)$, although all parameters (except for LA vortex volume) showed a significantly higher rescan variability than intra-observer variability $(\mathrm{p}<0.001)$. Finally, interval-scan variability was significantly higher than the technical rescan variability for LA stasis and LA mean velocity (both $\mathrm{p}<0.05$ ), but not for other flow parameters (all $\mathrm{p}>0.05$ ) (Table 1; Fig. 2).

Rescan and interval scan CVs, stratified for rhythm at the time of the scan (AF and SR), are shown for each parameter in Additional file 1: Table S6. We found no significant differences in rescan CVs by rhythm for any of the evaluated LA flow parameters (all $\mathrm{p}>0.05$ ). However, patients in AF displayed lower interval scan CV for stasis, mean velocity, and vortex volume (all $\mathrm{p}<0.05)$ compared with patients in SR. By contrast, no significant differences in interval scan CVs by rhythm were present for vorticity and peak velocity (all $\mathrm{p}>0.05$ ).

Finally, we conducted analysis to investigate whether changes in heart rate (HR) and blood pressure (BP) from scan 1a to scan 2 were associated with differences 


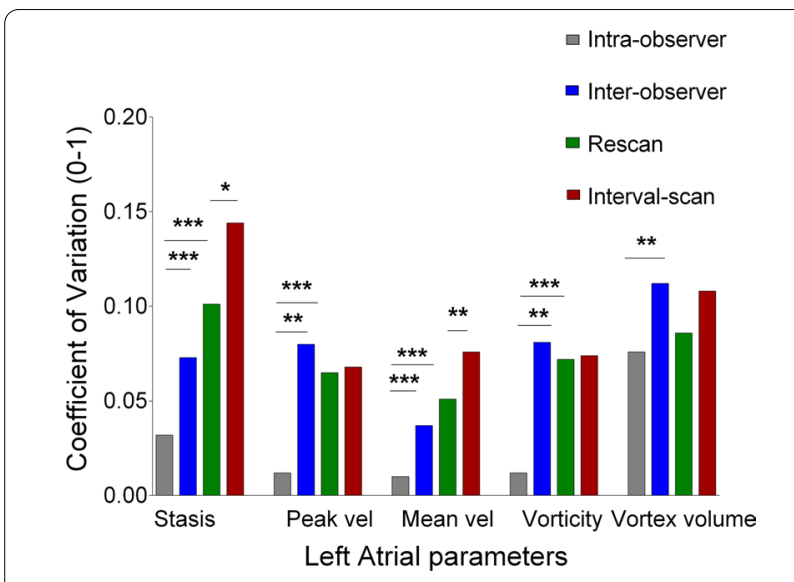

Fig. 2 Variability assessment of LA 4D flow parameters. Coefficients of variation (CV) for each flow parameter for intra-observer (grey), inter-observer (blue), rescan (green), and interval scan (red).CV are expressed as proportion (0-1). Statistically significant $p$ values are marked ${ }^{*}(<0.05)$, and smaller $p$ values are stratified by size ${ }^{* *} p<0.01$, $\left.{ }^{* * *} p<0.001\right)$

in interval scan variability (from scan 1a to scan 2) for any of the evaluated LA flow parameters. Interval-scan $C V s$ stratified by ' $\triangle H R \geq 10 \mathrm{bpm}$ vs $\Delta H R<10$ bpm' and ' $\triangle \mathrm{BP} \geq 10 \mathrm{mmHg}$ vs $\Delta \mathrm{BP}<10 \mathrm{mmHg}$ ' longitudinal changes are reported for each parameter in Additional file 1: Tables S7, S8. We found no significant differences in interval-scan CVs by BP for any of the evaluated flow parameters (all $\mathrm{p}>0.05$ ). However, $\geq 10 \mathrm{bpm}$ longitudinal changes in HR were associated with higher intervalscan variability for stasis $(C V=17 \%$ for $\geq 10 \mathrm{bpm}$ vs. $10 \%$ for $<10 \mathrm{bpm}$; $\mathrm{p}=0.024$ ); and were borderline associated with higher interval-scan variability for LA mean velocity $(\mathrm{CV}=8.5 \%$ for $\geq 10 \mathrm{bpm}$ vs. $6.0 \%$ for $<10 \mathrm{bpm}$; $\mathrm{p}=0.067$ ), see Additional file 1: Table S7. Notably, we found that longitudinal changes in HR correlated with changes in LA mean velocity and stasis $(\mathrm{p}=0.014$ and $\mathrm{p}=0.021$, respectively), with reduction of HR linked to lower mean velocity and increased stasis (Spearman's coefficient $=-0.29$ and -0.27 , respectively).

Inter-correlation among flow parameters is reported in Additional file 1: Table S9. Peak and mean velocity and stasis all display strong and significant inter-correlation (all $\mathrm{r} \geq 0.9$, all $\mathrm{p}<0.001$ ), whilst vorticity and vortex volume display moderate or mild correlation with the other flow parameters (all $\mathrm{r} \leq 0.8$, all $\mathrm{p}<0.01$ ).

\section{Discussion}

This is the first study investigating reproducibility and temporal variability of LA flow characteristic parameters, which is an important first step to determining their potential clinical applicability and use as imaging biomarkers. Using a large and comprehensive dataset, we quantified the degree of variability for several different flow parameters, and assessed potential contributing sources of errors including manual segmentation, subject positioning in the scanner, data acquisition, temporal changes, and rhythm at the time of the scan. Overall, LA peak velocity and vorticity are the most reproducible, temporally stable, and robust to rhythm differences and heart rate changes over time. Furthermore, these markers provide a non-invasive readout of LA haemodynamics that are potentially mechanistically relevant to thrombus formation and embolic stroke $[12,13]$.

\section{Reproducibility and temporal variability}

Of the evaluated LA flow parameters, peak and mean velocity and vorticity demonstrated the highest sameday scan-rescan reproducibility, comparable in magnitude to that for other widely-used cardiac imaging biomarkers such as LV end-diastolic volume and LVEF derived from CMR cine imaging, which are reported to have a scan-rescan $\mathrm{CV}$ of $5.7 \%$ and $6.1 \%$, respectively [33]. These results are also in keeping with prior data on reproducibility of 4D flow assessment of LV blood

Table 2 Reproducibility and temporal variability of flow patterns

\begin{tabular}{|c|c|c|c|c|c|c|}
\hline & $\operatorname{Scan}(N=84)$ & $\begin{array}{l}\text { Same-day rescan } \\
(N=84)\end{array}$ & $p$ value & Scan $(N=74)$ & $\begin{array}{l}\text { Interval scan } \\
(\mathrm{N}=74)\end{array}$ & $p$ value \\
\hline \multicolumn{7}{|l|}{ Systolic patterns } \\
\hline LA vortex pattern & $56(67)$ & $54(64)$ & 0.500 & $50(68)$ & $51(69)$ & $>0.999$ \\
\hline LA short-range vortex & $18(21)$ & $19(24)$ & $>0.999$ & $16(22)$ & $15(20)$ & $>0.999$ \\
\hline Others & $10(12)$ & $11(13)$ & $>0.999$ & $8(11)$ & $8(11)$ & $>0.999$ \\
\hline \multicolumn{7}{|l|}{ Diastolic patterns } \\
\hline LA vortex pattern & $46(55)$ & $46(55)$ & $>0.999$ & $37(50)$ & $41(55)$ & 0.454 \\
\hline LA short-range vortex & $11(13)$ & $10(12)$ & $>0.999$ & $11(15)$ & $9(12)$ & 0.625 \\
\hline Others & $27(31)$ & $28(33)$ & $>0.999$ & $26(35)$ & $24(32)$ & 0.815 \\
\hline
\end{tabular}

Data presented as $\mathrm{N}$ (\%). $\mathrm{p}$ values refer to paired comparison of proportions by McNemar's test 
flow components' volume ratio, where scan-rescan CVs ranged between 2.5 and $9.2 \%$ [34].

As expected, we found an overall incremental variability from intra-observer, to inter-observer and scan-rescan, also in keeping with previous studies [33]. Notably, we found that the variability attributable to human labelling of the LA (intra- and inter-observer CV) was only slightly lower than the scan-rescan variability for LA stasis, and was similar or greater than the scan-rescan variability for all the remaining LA flow parameters. This indicates a high degree of technical reproducibility of LA flow measurement, and is again consistent with CMR assessment of traditional imaging biomarkers such as LVEF [33].

As the ability to detect changes in LA flow parameters over time is of clinical interest, assessment of temporal (scan-interval scan) variability was also a key goal. We found that LA peak velocity and vorticity were the most stable parameters over time, in contrast to stasis and mean velocity, which displayed significantly higher temporal variability compared to their technical variability. Although LA vortex volume was also relatively stable over time, its variability was greater than that of both peak velocity and vorticity.

Importantly, the greater variability recorded with the interval scans over same-day rescan for LA stasis and mean velocity suggests a possible dependence on biological factors. In particular, we found a greater temporal variability in stasis and mean velocity in participants in SR, which was associated with longitudinal changes in heart rate (but not blood pressure). These data are consistent with a previously reported correlation between HR and 4D flow parameters in the LV [34], and are biologically plausible, given that changes in HR affect the dynamics of ventricular filling (i.e. the diastolic phase of the cardiac cycle), and therefore also affect atrial flow components when the mitral valve is open [35-37]. Previous experimental [38] and echocardiographic Doppler studies [37] demonstrated that a higher HR was associated with an increased atrial contribution to ventricular filling by (a) a predominant effect on the atrial flow integral, rather than peak velocities which remained stable [39], and (b) by affecting the passive filling phase, rather than the atrial booster phase [39]. In agreement with previous evidence [39], our data show that peak velocities are stable over time, but we showed for the first time that a reduction in HR is associated with increased LA stasis in SR. If confirmed in larger cohorts, this finding could provide novel insight into the finding that ivabradine (a negatively chronotropic agent) has been associated with an increased risk of incident AF [40] and ischemic stroke in patients with systolic heart failure in SR, in contrast to its beneficial effect on mortality and hospital re-admissions
[41]. Nevertheless, HR changes are likely to only partially explain the temporal variability observed in LA stasis, given the mild correlation observed, and other factors are likely to also be involved.

\section{Suitability for potential clinical application}

Low blood flow velocities (linked with activation of the coagulation cascade [2, 3]) and loss of normal coherent vortical flow (linked with platelet aggregation [15, 16]) have been associated with clinical stroke risk in recent validation 4D Flow CMR studies [12, 13]. Further studies assessing the association of LA 4D flow parameters in patients with paroxysmal or undiagnosed AF and thromboembolic events are needed. In order to design and plan such studies, the choice of which LA 4D flow biomarkers are most suitable to be tested for clinical application hinges on (a) reproducibility and temporal variability, (b) selection of those parameters that, in combination, provide complementary/additive information to each other, and (c) feasibility in terms of minimum sample size required for detection of a clinically meaningful difference. .

Of the LA 4D flow parameters evaluated in this study, stasis showed the highest technical and temporal variability, whilst peak velocity and vorticity were the most stable over time. Whilst adjustment for rhythm at the time of the scan is important for all 4D flow parameters to account for the dramatic rhythm-related changes in flow, it is particularly important for stasis, vortex volume, and mean velocity, which displayed higher temporal variability in SR than in AF. Further, the apparent sensitivity of stasis and mean velocity to $\mathrm{HR}$ is also relevant, particularly for longitudinal studies, whereas peak velocity and vorticity appear to be heart-rate independent.

Calculations of sample size for future clinical studies are not yet possible due to the lack of large longitudinal studies showing a meaningful significant difference based on prediction of embolic risk or AF. Further research is now needed to identify normal values for these 4D flow parameters in the general population, as well as to determine thresholds that may identify patients at higher risk of incident AF and/or embolic risk.

It is important to emphasise that, in principle, each parameter assesses different aspects of LA flow, and are all therefore potentially relevant markers of the overall propensity for thrombogenesis. Nevertheless, results from our study show that peak and mean velocity and stasis display a significant strong correlation with each other, implying they are likely to convey similar information. On the other hand, vorticity and vortex volume display only moderate or mild inter-correlation with the other parameters, making it more likely that they convey unique and complementary information. 
Taken together, our study shows that LA peak velocity and vorticity seem the most promising biomarkers to be tested for potential clinical application, since they are the most reproducible and stable over time, are robust to differences in heart rate and rhythm, and are likely to provide unique information. Both parameters are also mechanistically relevant, since low LA blood flow velocities are linked to activation of the coagulation cascade and increased red blood cell and plasma protein aggregation [2, 3], whilst loss of the normal coherent vortical LA flow pattern [14] is associated with platelet adhesion and aggregation $[15,16]$.

\section{Limitations}

Firstly, this study was undertaken at a single centre in a mixed cohort with non-consecutive recruitment and reproducibility assessment would need to be confirmed across multiple centres.

Secondly, in the current work, we focussed on the assessment of global LA flow characteristics and did not include the evaluation of LAA flow. Whilst assessment of local flow within the LAA or in other regions of the atrium is possible in principle [12] and clinically relevant, the post-processing tools for this are not widely available, and the reproducibility and temporal variability of LAA flow assessment are unknown. Once a postprocessing protocol for LAA flow analysis is available and demonstrated to be reproducible, futurestudies will be needed to clarify the value of LA analysis compared to LAA analysis, and the relative consequences for clinical applications. Thirdly, although the majority of patients underwent a follow-up scan, analysis of the differences in rescan vs. interval scans variability may be limited by the fact that the subjects who underwent scan 2 are a subset (86\%) of those who underwent scan 1. However, the $14 \%$ of patients who did not undergo an interval follow-up scan did not display significantly different rescan variability for any parameter compared to those who completed the follow-up visit (all $\mathrm{p}>0.05$ ).

In the presented work, we have not compared LA 4D flow parameters against transoesophageal echocardiography as a reference method. Another potential limitation is the use of four different $4 \mathrm{D}$ flow protocols (A, $\mathrm{B}, \mathrm{C}, \mathrm{D})$ and pulse sequences as specified in Additional file 1: methods. We used two similar retrospective protocols ('A' and 'B', one on each CMR system, both with spatial resolution $3.0 \times 3.0 \times 3.0 \mathrm{~mm}$ and retrospective gating, see further details in Additional file 1: methods) for $90 \%$ of the study population. In the remaining $10 \%$ of the population, we used 2 different sequences, which were part of our technical development at the beginning of the study: a prospective protocol (' $\mathrm{C}$ ' with the same parameters as protocol 'A' but prospectively gated) in 2 participants, and a separate retrospective protocol ' $\mathrm{D}$ ' (with higher spatial resolution: $2.4 \times 2.4 \times 2.5 \mathrm{~mm}$ ) in 7 participants (8\%). All follow-up scans were performed using the same CMR protocol as the respective baseline scan. We have also reported exploratory analysis of scanrescan coefficient of variation (CV) for each 4D Flow CMR protocol (available as Additional file 1: Table S10). Overall, the two main retrospective protocols displayed a scan-rescan CV in a similar range to those observed in the overall population.

Finally, 7 subjects ( $8 \%$ of those who underwent an interval scan, 4 in SR and 3 in AF) had changes to prescribed cardiovascular medications at the time of the interval scan compared to baseline. Whilst this could in principle confound the observed changes in LA stasis and mean velocity, there was no evidence of a statistical interaction between changes in these parameters and alterations to medication at follow-up $(p=0.833)$.

\section{Conclusions}

LA peak velocity and vorticity were the most reproducible and temporally stable novel LA 4D flow biomarkers, and are robust to changes in heart rate, blood pressure, and differences in heart rhythm.

\section{Abbreviations}

4D flow: Four dimensional flow; AF: Atrial fibrillation; BP: Blood pressure; bSSFP: Balanced steady-state free precession; Cl: Confidence interval; CMR: Cardiovascular magnetic resonance; CV: Coefficient of variation; ECG: Electrocardiogram; HR: Heart rate; LA: Left atrium; LAA: Left atrial appendage; LAEF: Left atrial ejection fraction; LoA: Limits of agreement; LV: Left ventricle/left ventricular; LVEF: Left ventricular ejection fraction; ICC: Intra-class correlation coefficient; SD: Standard deviation; SR: Sinus rhythm.

\section{Supplementary Information}

The online version contains supplementary material available at https://doi. org/10.1186/s12968-021-00729-0.

Additional file 1: Supplementary material.

Additional file 2: Video S1.

\section{Acknowledgements}

The authors gratefully acknowledge the research nurses within OCMR and AVIC for their help and support during the study visits.

\section{Authors' contributions}

MS designed the study and obtained regulatory approvals, led the technical development of 4D Flow post-processing tools, recruited all participants, organized the data management for the purpose of blinded analyses and for the unblinding phase, undertook statistical analysis, and prepared the manuscript. ATH and MS designed the Matlab-based in-house software which was coded by ATH. AF, PM, and MS scanned all the participants. AVE was the study statistician. GPA and AS were the blinded observers. AS provided intellectual contribution to development of flow patterns and helped with data management for the output from Matlab post-processing software. RSW contributed substantially to the conception, design, funding, and oversight of the study, and led critical revision of the manuscript. ATH, VMF, SN, and BC provided key 
intellectual contributions to study design and data interpretation. All authors participated in manuscript revision including reading the final manuscript. All authors read and approved the final manuscript.

\section{Funding}

The study was funded by the National Institute for Health Research (NIHR) Biomedical Research Centre (BRC) based at Oxford University Hospitals Foundation Trust at the University of Oxford, UK. MS and GPA are funded by the NIHR Oxford BRC. MS acknowledges support from a competitive scholarship for young cardiologists awarded by the Italian Society of Cardiology and funded via MSD ITALIA-MERCK SHARP \& DOHME CORPORATION. VMF and $B C$ are funded by the British Heart Foundation (BHF). VMF, ATH, RSW, and MS acknowledge support from the BHF Centre of Research Excellence, Oxford.

\section{Availability of data and materials}

The Matlab-based in-house software designed by ATH and MS (MATLAB R2015 8.6.0.267246; Mathworks, Natick, Massachusetts, USA), has made available on the Oxford University Research Archive (ORA) platform (https://doi. org/10.5287/bodleian:ey4ovzdbB). The datasets generated and analysed during the current study are not publically available due to regulatory restrictions. Data that support the findings of this study are available from the corresponding author upon reasonable request.

\section{Ethics approval and consent to participate}

This study was approved by the local research ethics committee: Yorkshire \& The Humber-Leeds East Research Ethics Committee (REC reference: 18/ $\mathrm{YH} / 0096)$. All patients gave written informed consent. Healthy volunteers recruited under an ethically-approved departmental technical development protocol provided verbal consent (as specified in the protocol).

\section{Consent for publication}

Informed consent was obtained from all participants for inclusion of their data in publications.

\section{Competing interests}

$B C$ is receiving support in kind from Roche Diagnostics (blood assays) and iRhythm (ECG monitors) in clinical studies on atrial fibrillation. RW has received honoraria and/or travel assistance from Biosense Webster, Bayer, Boston Scientific and Abbott. All authors report no conflicts of interest in relation to this work.

\section{Author details \\ 1 Division of Cardiovascular Medicine, Radcliffe Department of Medicine, John Radcliffe Hospital, University of Oxford, West Wing, Headley Way, Oxford, UK. ${ }^{2}$ The University of Oxford Centre for Clinical Magnetic Resonance Research (OCMR), Oxford, UK. ${ }^{3}$ The University of Oxford Acute Vascular Imaging Centre (AVIC), Oxford, UK. ${ }^{4}$ Department of Population Health, CTSU Nuffield Univer- sity of Oxford, Oxford, UK.}

Received: 11 June 2020 Accepted: 3 February 2021

Published online: 22 March 2021

\section{References}

1. Feigin VL, Norrving B, Mensah GA. Global burden of stroke. Circ Res. 2017;120:439-48.

2. Watson T, Shantsila E, Lip GY. Mechanisms of thrombogenesis in atrial fibrillation: Virchow's triad revisited. Lancet. 2009;373:155-66.

3. Fatkin D, Kelly RP, Feneley MP. Relations between left atrial appendage blood flow velocity, spontaneous echocardiographic contrast and thromboembolic risk in vivo. J Am Coll Cardiol. 1994;23:961-9.

4. Handke M, Harloff A, Hetzel A, Olschewski M, Bode C, Geibel A. Left atrial appendage flow velocity as a quantitative surrogate parameter for thromboembolic risk: determinants and relationship to spontaneous echocontrast and thrombus formation - a transesophageal echocardiographic study in 500 patients with cerebral ischemia. J Am Soc Echocardiogr. 2005;18:1366-72.

5. Stoddard MF, Dawkins PR, Prince CR, Ammash NM. Left atrial appendage thrombus is not uncommon in patients with acute atrial fibrillation and a recent embolic event: a transesophageal echocardiographic study. J Am Coll Cardiol. 1995;25:452-9.

6. Shrestha NK, Moreno FL, Narciso FV, Torres L, Calleja HB. Two-dimensional echocardiographic diagnosis of left-atrial thrombus in rheumatic heart disease. A clinicopathologic study. Circulation. 1983;67:341-7.

7. Blackshear JL, Odell JA. Appendage obliteration to reduce stroke in cardiac surgical patients with atrial fibrillation. Ann Thorac Surg. 1996;61:755-9.

8. Kamel H, Okin PM, Elkind MS, ladecola C. Atrial fibrillation and mechanisms of stroke: time for a new model. Stroke. 2016;47:895-900.

9. Friberg L, Rosenqvist M, Lip GY. Evaluation of risk stratification schemes for ischaemic stroke and bleeding in 182678 patients with atrial fibrillation: the Swedish Atrial Fibrillation cohort study. Eur Heart J. 2012;33:1500-10.

10. Ntaios G, Lip GY, Makaritsis K, et al. CHADS(2), CHA(2)S(2)DS(2)-VASC, and long-term stroke outcome in patients without atrial fibrillation. Neurology. 2013;80:1009-17.

11. Dyverfeldt P, Bissell M, Barker AJ, et al. 4D flow cardiovascular magnetic resonance consensus statement. J Cardiovasc Magn Reson. 2015;17:72.

12. Markl M, Lee DC, Furiasse N, et al. Left atrial and left atrial appendage $4 \mathrm{D}$ blood flow dynamics in atrial fibrillation. Circ Cardiovasc Imaging. 2016;9:e004984.

13. Garcia J, Sheitt H, Bristow MS, et al. Left atrial vortex size and velocity distributions by $4 \mathrm{D}$ flow MRI in patients with paroxysmal atrial fibrillation: associations with age and CHA2 DS2 -VASc risk score. Journal of magnetic resonance imaging: JMRl; 2019.

14. Kilner PJ, Yang GZ, Wilkes AJ, Mohiaddin RH, Firmin DN, Yacoub $\mathrm{MH}$. Asymmetric redirection of flow through the heart. Nature. 2000;404:759-61.

15. Yazdani A, Li H, Humphrey JD, Karniadakis GE. A general sheardependent model for thrombus formation. PLoS Comput Biol. 2017;13:e1005291.

16. Gulan U, Saguner A, Akdis D, et al. Investigation of atrial vortices using a novel right heart model and possible implications for atrial thrombus formation. Sci Rep. 2017;7:16772.

17. Healey JS, Alings M, Ha A, et al. Subclinical atrial fibrillation in older patients circulation. 2017;136:1276-83.

18. Wijesurendra RS, Liu A, Eichhorn C, et al. Lone atrial fibrillation is associated with impaired left ventricular energetics that persists despite successful catheter ablation. Circulation. 2016;134:1068-81.

19. Wijesurendra RS, Liu A, Notaristefano F, et al. Myocardial perfusion is impaired and relates to cardiac dysfunction in patients with atrial fibrillation both before and after successful catheter ablation. J Am Heart Assoc. 2018;7:e009218.

20. Norris DG, Hutchison JM. Concomitant magnetic field gradients and their effects on imaging at low magnetic field strengths. Magn Reson Imaging. 1990;8:33-7.

21. Purvis $L A B$, Clarke WT, Biasiolli L, Valkovic $L$, Robson MD, Rodgers $C T$. OXSA: an open-source magnetic resonance spectroscopy analysis toolbox in MATLAB. PLOS ONE. 2017;12:e0185356.

22. Loecher M, Schrauben E, Johnson KM, Wieben O. Phase unwrapping in 4D MR flow with a 4D single-step laplacian algorithm. J Magn Reson Imaging. 2016:43:833-42.

23. Caprihan A, Benitez-Read SAA,E. Flow-velocity imaging from linear regression of phase images with techniques for reducing eddy-current effects. J Magn Reson. 1990;90:71-89.

24. Bustamante M, Gupta V, Carlhall CJ, Ebbers T. Improving visualization of $4 \mathrm{D}$ flow cardiovascular magnetic resonance with four-dimensional angiographic data: generation of a 4D phase-contrast magnetic resonance CardioAngiography (4D PC-MRCA). J Cardiovasc Magn Reson. 2017;19:47.

25. Markl M, Carr M, Ng J, et al. Assessment of left and right atrial 3D hemodynamics in patients with atrial fibrillation: a 4D flow MRI study. Int J Cardiovasc Imaging. 2016;32:807-15.

26. Elbaz MS, Calkoen EE, Westenberg JJ, Lelieveldt BP, Roest AA, van der Geest RJ. Vortex flow during early and late left ventricular filling in normal subjects: quantitative characterization using retrospectively-gated 4D flow cardiovascular magnetic resonance and three-dimensional vortex core analysis. J Cardiovasc Magn Reson. 2014;16:78.

27. Suwa K, Saitoh T, Takehara Y, et al. Characteristics of intra-left atrial flow dynamics and factors affecting formation of the vortex flow-analysis with 
phase-resolved 3-dimensional cine phase contrast magnetic resonance imaging. Circ J. 2015;79:144-52.

28. Eriksson J, Carlhall CJ, Dyverfeldt P, Engvall J, Bolger AF, Ebbers T. Semiautomatic quantification of $4 \mathrm{D}$ left ventricular blood flow. J Cardiovasc Magn Reson. 2010;12:9.

29. Fyrenius A, Wigstrom L, Ebbers T, Karlsson M, Engvall J, Bolger AF. Three dimensional flow in the human left atrium. Heart. 2001;86:448-55.

30. Ong F, Uecker $M$, Tariq U, et al. Robust 4D flow denoising using divergence-free wavelet transform. Magn Reson Med. 2015;73:828-42.

31. Pal PZZ, Lin BA, Dione D, Sinusas AJ, Sampath S. Flow vortex quantification in the left atrium. J Cardiovasc Magn Reson. 2012;14(Suppl 1):W23.

32. Kim WY, Walker PG, Pedersen EM, et al. Left ventricular blood flow patterns in normal subjects: a quantitative analysis by three-dimensional magnetic resonance velocity mapping. J Am Coll Cardiol. 1995;26:224-38,

33. Bhuva N, Bulluck H, Captur G, et al. A multicenter, scan-rescan, human and machine learning CMR study to test generalizability and precision in imaging biomarker analysis. Circ Cardiovasc Imaging. 2019;12:e009214.

34. Stoll VM, Loudon M, Eriksson J, et al. Test-retest variability of left ventricular 4D flow cardiovascular magnetic resonance measurements in healthy subjects. J Cardiovasc Magn Reson. 2018:20:15.

35. Nolan SP, Dixon SH Jr, Fisher RD, Morrow AG. The influence of atrial contraction and mitral valve mechanics on ventricular filing. A study of instantaneous mitral valve flow in vivo. Am Heart J. 1969;77:784-91.

36. Skinner NS Jr, Mitchell JH, Wallace AG, Sarnoff SJ. Hemodynamic effects of altering the timing of atrial systole. Am J Physiol. 1963;205:499-503.
37. Harrison MR, Clifton GD, Pennell AT, DeMaria AN. Effect of heart rate on left ventricular diastolic transmitral flow velocity patterns assessed by Doppler echocardiography in normal subjects. Am J Cardiol. 1991;67:622-7

38. Ishida Y, Meisner JS, Tsujioka K, et al. Left ventricular filling dynamics: influence of left ventricular relaxation and left atrial pressure. Circulation. 1986;74:187-96.

39. Iliceto S, D'Ambrosio G, Marangelli V, Amico A, Di Biase M, Rizzon P. EchoDoppler evaluation of the effects of heart rate increments on left atrial pump function in normal human subjects. Eur Heart J. 1991;12:345-51.

40. Martin RI, Pogoryelova O, Koref MS, Bourke JP, Teare MD, Keavney BD. Atrial fibrillation associated with ivabradine treatment: meta-analysis of randomised controlled trials. Heart. 2014;100:1506-10.

41. Koki Nakanishi MRDT, Qian M, Thompson JLP, Labovitz AJ, Mann DL, Sacco RL, Pullicino PM, Freudenberger RS, Teerlink JR, Graham S, Gregory YH, Lip B, Levin JP, Mohr R, Buchsbaum CJ, Estol DJL, Ponikowski P, Anker SD, Homma S, WARCEF Investigators. Resting heart rate and ischemic stroke in patients with heart failure. Cerebrovasc Dis. 2017:44:43-50.

\section{Publisher's note}

Springer Nature remains neutral with regard to jurisdictional claims in published maps and institutional affiliations.
Ready to submit your research? Choose BMC and benefit from:

- fast, convenient online submission

- thorough peer review by experienced researchers in your field

- rapid publication on acceptance

- support for research data, including large and complex data types

- gold Open Access which fosters wider collaboration and increased citations

- maximum visibility for your research: over 100M website views per year

At BMC, research is always in progress.

Learn more biomedcentral.com/submissions 